Article

\title{
Omega-3 Fatty Acids Survey in Men under Active Surveillance for Prostate Cancer: from Intake to Prostate Tissue Level
}

\author{
Hanane Moussa ${ }^{1}$, Molière Nguile-Makao ${ }^{1}$, Karine Robitaille ${ }^{1}$, Marie-Hélène Guertin ${ }^{1}$, \\ Janie Allaire ${ }^{2}$, Jean-François Pelletier ${ }^{1}$, Xavier Moreel ${ }^{1,3}{ }^{\text {, Nikunj Gevariya }}{ }^{1}$, Caroline Diorio ${ }^{1}$, \\ Patrice Desmeules ${ }^{3,4}$, Bernard Têtu ${ }^{1,4}$, Benoît Lamarche ${ }^{2} \mathbb{D}$, Pierre Julien ${ }^{5}$ and \\ Vincent Fradet 1,2,* \\ 1 Oncology Axis, Centre de recherche du CHU de Québec - Université Laval, QC G1R 3S1, Canada \\ 2 Institute of Nutrition and Functional Foods (INAF), Université Laval, QC G1V 0A6, Canada \\ 3 Centre de recherche de l'Institut Universitaire de Cardiologie et Pneumologie de Québec, Université Laval, \\ QC G1V 4G5, Canada \\ 4 Department of Pathology, CHU de Québec - Hôpital Saint-Sacrement, QC G1S 4L8, Canada \\ 5 Endocrinology and Nephrology Axis, Centre de recherche du CHU de Québec - Université Laval, \\ QC G1V 4G2, Canada \\ * Correspondence: Vincent.Fradet@fmed.ulaval.ca; Tel.: +1-418-525-4444 (ext. 16841); Fax: +1-418-691-3154
}

Received: 15 June 2019; Accepted: 12 July 2019; Published: 16 July 2019

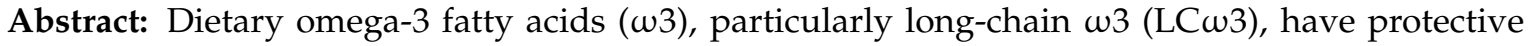
effects against prostate cancer (PCa) in experimental studies. Observational studies are conflicting, possibly because of the biomarker used. This study aimed at evaluating associations between grade reclassification and $\omega 3$ levels assessed in prostatic tissue, red blood cells (RBC), and diet. We conducted a validation cross-sectional study nested within a phase II clinical trial. We identified 157 men diagnosed with low-risk PCa who underwent a first active surveillance repeat prostate biopsy session. Fatty acid (FA) intake was assessed using a food frequency questionnaire and their levels measured in prostate tissue and RBC. Associations were evaluated using logistic regression. At first repeat biopsy session, 39 (25\%) men had high-grade PCa (grade group $\geq 2$ ). We found that high LC $\omega 3$-eicosapentaenoic acid (EPA) level in prostate tissue (odds ratio (OR) 0.25; 95\% (confidence interval (CI) 0.08-0.79; $p$-trend =0.03) was associated with lower odds of high-grade PCa. Similar results were observed for LC $\omega 3$ dietary intake (OR 0.30; 95\% CI 0.11-0.83; $p$-trend $=0.02$ ) but no association for RBC. LCw3-EPA levels in the target prostate tissue are inversely associated with high-grade PCa in men with low-risk PCa, supporting that prostate tissue FA, but not RBC FA, is a reliable biomarker of PCa risk.
\end{abstract}

Keywords: Omega-3 fatty acids; Eicosapentaenoic acid; Gleason score; High-grade prostate cancer; Active surveillance

\section{Introduction}

The worldwide difference in prostate cancer (PCa) incidence highlights an important role of lifestyle in PCa [1-4]. Dietary fatty acid (FA) intake is one dietary factor thought to impact PCa development. Interestingly, Inuit people have a very low incidence of PCa and pre-malignant lesion at autopsy, which may be attributed to their traditional diet rich in omega-3 FA $(\omega 3)[5,6]$. In addition, preclinical and clinical experimental studies support that high $\omega 3$ intake have protective effects against PCa, likely via their anti-inflammatory properties [7]. Animal studies showed that $\omega 3$, particularly long-chain $\omega 3$ (LC $\omega 3$ ), suppress neoplastic transformation, angiogenesis, and tumor cell growth $[8,9]$. 
A prospective randomized trial with fish oil supplementation showed that LC $\omega 3$ decrease PCa cell proliferation in men undergoing radical prostatectomy [10,11].

Epidemiological studies provide mixed results regarding associations between FA intake and PCa. Some studies showed that a high level of $\omega 3$ is inversely associated with PCa risk [12-16], while other studies demonstrated null [17-21] or positive associations [22-24]. These studies used various methods to evaluate FA intake, such as food frequency questionnaires (FFQ) and FA composition of plasma circulating lipids or of red blood cell (RBC) membranes. Most of the studies evaluating the association between $\omega 3$ and PCa were based on FFQ [12-15,19,23,25,26], while fewer studies were based on FA levels in plasma or in RBC membranes [16,18,20-22,24]. The FA composition of RBC is thought to be more reliable than plasma, as it reflects the last 3-month diet [27]. To our knowledge, only one previous study was based on FA levels in the prostate tissue [28]. In this cohort of 48 men with low-risk PCa, we measured the level of eicosapentaenoic acid (EPA), a LC $\omega 3$ subtype, directly in prostatic tissue, and found that it was inversely associated with reclassification to high-grade disease. This association was not observed in RBC, suggesting that information bias may be present when considering only blood FA measures.

We undertook a validation study using the same methodology. We hypothesized that $\omega 3$ intake, and particularly LC $\omega 3$ levels in the target prostate tissue, are inversely associated with high-grade PCa during active surveillance. The $\omega 3$ can be assessed through food intake, directly in circulating RBC or in target tissue such as prostate. In this study, we aimed at evaluating the associations between $\omega 3$ status assessed through different measurements and high-grade $\mathrm{PCa}$, or grade reclassification, in men under active surveillance for a low-risk PCa.

\section{Materials and Methods}

\subsection{Study Population}

This study is a cross-sectional study of 189 men recruited for a phase II randomized controlled trial. Men were recruited at the time of a low-risk PCa diagnosis for which active surveillance was elected. Low-risk PCa was defined as pathologically confirmed grade group 1 , with $<6$ positive biopsy cores out of $12,<3$ positive sextants, a clinical stage $\leq$ T2a, and a prostate-specific antigen (PSA) level $<15 \mathrm{ng} / \mathrm{mL}$ if prostate volume was $>30 \mathrm{~mL}$, or a PSA $<10 \mathrm{ng} / \mathrm{mL}$ otherwise. Patients were excluded if they were taking a $5 \alpha$-reductase inhibitor (5-ARI) drug, $\omega 3$ supplements, or if they had received radiotherapy or chemotherapy. The ongoing randomized trial assesses the effects on prostate tissue biomarkers of a dietary intervention versus dutasteride, a 5-ARI drug. The dietary intervention aims at increasing intake of LC $\omega 3$ while decreasing $\omega 6$ FA and trans-fat intake.

The current cross-sectional study investigates how FA levels are associated with a high-grade $\mathrm{PCa}$ (grade group $\geq 2$ ) at the first repeat biopsy, which was performed for randomization into the trial. A planned analysis of the first 48 patients, which amounted to about one-fourth of all patients recruited, has already been published [28]. We present here the data at baseline for the complete study population. From 189 men recruited for this study, 176 were eligible according to exclusion criteria listed in Figure 1. Men were included if information was available for FA intake obtained from FFQ, as well as FA levels in RBC and in prostate tissue $(n=157)$.

All patients provided written informed consent to participate in the study. The protocol is registered to clinicaltrials.gov (NCT01653925). The Centre Hospitalier Universiatire (CHU) de Québec-Université Laval ethics board approved the trial and the current study (2012-466). 
Low-risk prostate cancer

Assessed for eligibility $(\mathrm{n}=189)$

\begin{tabular}{|c|c|}
\hline \multirow{2}{*}{$\downarrow$} & $\begin{array}{l}\quad \text { Ineligible }(n=13) \\
\text { - Use of Avodart }(n=4) \\
\text { - Use of omega-3 supplements }(n=5) \\
\text { - Retracted consent }(n=2) \\
\text { - Central pathology review of diagnostic biopsy to } \\
\text { Gleason } 7(n=1) \text { or to absence of cancer }(n=1)\end{array}$ \\
\hline & \\
\hline \multicolumn{2}{|c|}{ First repeat prostate biopsy session $(n=176)$} \\
\hline$\downarrow$ & $\begin{array}{l}\text { - Incomplete Web-FFQ or lack of information on } \\
\text { FA in prostate tissue or in RBC }(n=13) \\
\text { - Error measurement }(n=2) \\
\text { - Missing adjustment variables from incomplete } \\
\text { questionnaires }(n=4)\end{array}$ \\
\hline \multicolumn{2}{|c|}{$\begin{array}{l}\text { Included for analysis } \\
\qquad(n=157)\end{array}$} \\
\hline
\end{tabular}

Figure 1. Flow chart of selection for study subjects. 189 men diagnosed with low-grade prostate cancer (PCa) and electing an active surveillance program were recruited for this study. After several months, men underwent a new prostate biopsy session (first repeat, or confirmatory biopsy) to assess PCa grade. Note: Web-FFQ = validated Food Frequency Questionnaire online; FA = Fatty Acids; RBC = Red Blood Cells.

\subsection{Data Collection}

\subsubsection{PCa Grade}

Following the initial diagnosis of low-risk $\mathrm{PCa}$, patients underwent a first repeat (confirmatory) biopsy session performed by the same ultrasonographist (VF), which was planned within 6 months (range 2-14 months) after initial diagnosis. Central pathology review of all biopsy cores was evaluated using the recent WHO-endorsed Gleason grading system, as proposed by Epstein [29,30]. High-grade PCa was defined as Gleason score $\geq 7$, which included grade group 2 (Gleason 3+4) or greater.

\subsubsection{FA Profiles}

FA levels in prostatic tissue and RBC: At first repeat biopsy session, additional prostate biopsy cores were taken in normal peripheral zones of the prostate presenting no prostate nodule, no ultrasound abnormality, and within a sextant region where the initial diagnostic biopsy cores were negative. These biopsy cores were dedicated to research and were immediately placed in cold Hank's Balanced Salt Solution (HBSS; Invitrogen), frozen in dry ice, and stored at $-80^{\circ} \mathrm{C}$.

Blood samples were collected into dipotassium ethylenediaminetetraacetic acid (K2EDTA)-containing Vacutainer tubes (Becton Dickinson) on the morning of the first repeat biopsy session, after an overnight fast, and immediately placed on ice. Within $1 \mathrm{~h}$, plasma, buffy coat, and RBC were separated and isolated by centrifugation $\left(2500 \mathrm{~g}, 15 \mathrm{~min}, 4^{\circ} \mathrm{C}\right)$. Isolated fractions were stored at $-80^{\circ} \mathrm{C}$.

FA profiles of normal prostate biopsies and RBC were determined by gas chromatography after extraction of total lipids, as previously described [28,31,32]. The reliability of repeat measurements of FA profiles in prostatic tissue by gas chromatography were previously demonstrated [28]. FA profiles 
in RBC is expressed as percentage of total FA, while in prostatic tissue as percentage of total FA as well as in milligrams of FA per gram of tissue.

FA intake: Patients were asked to complete a validated quantitative, web-based, self-administered FFQ online (web-FFQ) within a few days of the first repeat biopsy session to assess food intake over the last month. This web-FFQ contains standardized photographs of most food items, to help the participant quantify portion sizes, and was developed and validated specifically for Quebec citizens, who constitute the totality of our study population [33]. We also previously validated ability of this web-FFQ to assess $\omega 3$ intake specifically in PCa patients [34].

\subsection{Confounding Variables}

Anthropometric measures were taken according to standardized procedures by research personnel trained specifically for this assessment. Information pertaining to cancer risk factors, such as age, education level, smoking status, medical history, and physical activity, was also collected. Physical activity was measured using the validated Godin-Shephard Leisure-Time Physical Activity Questionnaire [35]. In this questionnaire, duration and intensity components are scored, which allows classification of individuals into three validated categories: active (PA score $\geq 24$ ), moderately active $(14 \leq$ PA score $<24)$ and inactive (PA score $<14)[36]$.

\subsection{Statistical Analysis}

Descriptive statistics for low- and high-grade PCa groups at first repeat biopsy were compared using Wilcoxon test for continuous variables and using chi-squared test for categorical variables.

To estimate associations between FA status (assessed either in prostate tissue, RBC, or diet) and reclassification to high-grade PCa at first repeat biopsy, we categorized FA in tertiles. We fitted logistic regression models to estimate odds ratios (OR) and 95\% confidence intervals (95\% CI) of high-grade disease across FA tertiles, relative to the lowest tertile. Models were adjusted for potential confounding factors identified from the literature and that are potentially associated with PCa grade $(p \leq 0.30$ in the multivariable analysis): waist circumference (continuous), PSA (continuous), alcohol (continuous), and physical activity (active, moderately active, and inactive) [36,37]. In addition, age (continuous) and total energy intake (continuous) were included in all models independently of p-values. We performed sensitivity analyzes by adjusting for body mass index (BMI) instead of waist circumference, for prostate volume, and also for education. These sensitivity analyses did not materially change the results. We also conducted a trend test ( $p$-trend) by treating the median of tertiles as a continuous variable in multivariable regression models. In order to explore the possibility of residual confounding by using tertiles, we categorized more finely the prostate tissue EPA levels according to their observed distribution (Supplementary Figure S1), showing a group at the inferior limit of sensitivity, a middle group split in half, and high outliers $(0 ; 0-0.2 ; 0.2-0.4 ;>0.4)$. We tested the dose-response effect by using the Mantel-Haenszel test, as is was not possible to normalize the distribution of prostate tissue EPA, precluding linear modeling. Finally, we calculated correlations between FA from web-FFQ, RBC membranes, and prostate tissue using partial Spearman correlations.

All statistical analyses were performed using SAS version 9.4. All tests were two-sided and $p<0.05$ was considered as statistically significant. In order to evaluate the possibility of selection bias, we compared the characteristics of included and excluded participants.

\section{Results}

We identified 157 men meeting inclusion criteria. At first repeat—or confirmation—biopsy, 39 men were reclassified to high-grade PCa and 118 had low-grade disease. Characteristics of the study population are summarized in Table 1 . The mean age of patients was 61 years $( \pm 7)$ and the mean PSA was $5.0 \mathrm{ng} / \mathrm{mL}( \pm 2.7)$. Of those men, $43 \%$ had a university degree and $38 \%$ had never smoked. BMI higher than $30 \mathrm{Kg} / \mathrm{m}^{2}$ was observed in $23 \%$ of participants. The characteristics of the included and excluded patients were similar, except for age (Supplementary Table S1). 
Table 1. Characteristics of the study subjects at the first repeat biopsy session.

\begin{tabular}{|c|c|c|c|c|}
\hline Variables & $\begin{array}{c}\text { Total } \\
(n=157)\end{array}$ & $\begin{array}{l}\text { Low-Grade } \\
\qquad(n=118)\end{array}$ & $\begin{array}{l}\text { High-Grade } \\
\quad(n=39)\end{array}$ & $p$-Value ${ }^{*}$ \\
\hline \multicolumn{5}{|l|}{ Age (y) } \\
\hline Mean \pm SD & $61.3 \pm 7.4$ & $61.3 \pm 7.2$ & $61.2 \pm 8.2$ & \multirow[t]{2}{*}{0.84} \\
\hline Median (Q1-Q3) & $62.0(56.0-67.0)$ & $62.0(56.0-67.0)$ & $62.0(56.0-67.0)$ & \\
\hline \multicolumn{5}{|l|}{ PSA (ng/mL) } \\
\hline Mean \pm SD & $5.0 \pm 2.7$ & $5.04 \pm 2.9$ & $5.05 \pm 2.05$ & \multirow{2}{*}{0.26} \\
\hline Median (Q1-Q3) & $4.7(3.3-6.0)$ & $4.6(3.0-6.2)$ & $4.8(4.0-5.9)$ & \\
\hline \multicolumn{5}{|l|}{ Waist Circumference (cm) } \\
\hline Mean \pm SD & $90.0 \pm 10.2$ & $95.2 \pm 9.3$ & $98.5 \pm 12.5$ & \multirow[t]{2}{*}{0.42} \\
\hline Median (Q1-Q3) & $96.0(89.0-102.0)$ & $95.0(88.0-101.0)$ & $96.0(89.0-105.0)$ & \\
\hline \multicolumn{5}{|l|}{ BMI Kg/m², n (\%) } \\
\hline$<25$ & $44(28.39)$ & $35(29.66)$ & $9(24.32)$ & \multirow{3}{*}{0.26} \\
\hline $25-30$ & $76(49.03)$ & $60(50.85)$ & $16(43.24)$ & \\
\hline$>30$ & $35(22.58)$ & $23(19.49)$ & $12(32.43)$ & \\
\hline \multicolumn{5}{|c|}{ Education level attained, $n(\%)$} \\
\hline Secondary school or less & $46(29.30)$ & $31(26.27)$ & $15(38.46)$ & \multirow{3}{*}{0.34} \\
\hline Postsecondary diploma & $44(28.03)$ & $34(28.81)$ & $10(25.64)$ & \\
\hline University degree & $67(42.68)$ & $53(44.92)$ & $14(35.90)$ & \\
\hline \multicolumn{5}{|l|}{ Smoking status, $n(\%)$} \\
\hline Current smoker & $10(6.37)$ & $7(5.93)$ & $3(7.69)$ & \multirow{3}{*}{0.91} \\
\hline Former smoker & $88(56.05)$ & $66(55.93)$ & $22(56.41)$ & \\
\hline Never & $59(37.58)$ & $45(38.14)$ & $14(35.90)$ & \\
\hline \multicolumn{5}{|l|}{ Physical activity score, $n(\%)$} \\
\hline Active & $94(59.87)$ & $74(62.71)$ & $20(51.28)$ & \multirow{3}{*}{0.33} \\
\hline Moderately active & $13(8.28)$ & $8(6.78)$ & $5(12.82)$ & \\
\hline Inactive & $50(31.85)$ & $36(30.51)$ & $14(35.90)$ & \\
\hline
\end{tabular}

NOTE: In this study, 157 men diagnosed with low-risk prostate cancer (PCa) and managed under active surveillance underwent a first repeat biopsy session. At the first repeat biopsy session, 39 men out of 157 had a high-grade PCa (grade group $\geq 2$ ). Note: ${ }^{*}=p$-values were obtained using the Wilcoxon test for continuous variables and chi-2 test for categorical variables. $\mathrm{SD}=$ standard deviation; $\mathrm{Q} 1$ = lower quartile; $\mathrm{Q} 3=$ upper quartile; $\mathrm{PSA}$ = prostate-specific antigen; $\mathrm{BMI}=$ body mass index.

Table 2 presents FA dietary intake, FA profiles in RBC, and in prostatic tissue at the first repeat biopsy session. The mean caloric intake was $2500 \mathrm{kcal}( \pm 759)$. Participants had an average daily intake of $\omega 3$ and $\mathrm{LC} \omega 3$ of $2.17 \mathrm{~g}( \pm 0.90)$ and $0.41 \mathrm{~g}( \pm 0.35)$, respectively. The mean $\omega 3 / \omega 6$ ratio from dietary intake was $0.15( \pm 0.05)$ and was borderline higher in low-grade PCa group $(p=0.05)$. This difference was significantly reflected in prostate tissue $(p<0.01)$. Finally, LC $\omega 3$ subtype EPA level in prostatic tissue was higher in low-grade PCa group $(p=0.01)$.

Associations between FA levels in prostate tissue and reclassification to high-grade PCa are presented in Table 3. FA levels in prostate tissue are associated with high-grade PCa. Compared to men in the lowest tertile of EPA level in prostatic tissue, men in the highest tertile had a decreased risk of high-grade $\mathrm{PCa}(\mathrm{OR} 0.25 ; 95 \% \mathrm{CI} 0.08-0.79 ; p$-trend $=0.03)$. Weaker and stronger associations were observed, respectively, for the LC $\omega 3 / \omega 6$ ratio and $\omega 3 / \omega 6$ ratio measured in prostatic tissue (OR $0.38 ; 95 \%$ CI $0.14-1.00 ; p$-trend $=0.05$, and OR $0.20 ; 95 \%$ CI 0.07-0.59; $p$-trend $<0.01$, respectively). Table 4 shows the analysis with finer categories of EPA measured in prostate tissue (Supplementary Figure S1), in which we found a significant dose-response association between EPA and high-grade $(p$-trend $<0.01)$. 
Table 2. Fatty acid intake, fatty acid profiles of RBC membranes, and of prostate tissue stratified by prostate cancer grade.

\begin{tabular}{|c|c|c|c|c|c|}
\hline Method & Variables & $\begin{array}{c}\text { Total } \\
n=157\end{array}$ & $\begin{array}{c}\text { Low-Grade } \\
n=118\end{array}$ & $\begin{array}{c}\text { High-Grade } \\
\quad n=39\end{array}$ & $p$-Value ${ }^{\S}$ \\
\hline \multirow{36}{*}{ Web-FFQ * } & Energy (kcal) & & & & \multirow{3}{*}{0.23} \\
\hline & Mean \pm SD & $2496 \pm 759$ & $2468 \pm 755$ & $2583 \pm 775$ & \\
\hline & Median (Q1-Q3) & 2449 (1901-2952) & $2416(1871-2869)$ & 2604 (1991-2975) & \\
\hline & Alcohol (g) & & & & \multirow{3}{*}{0.42} \\
\hline & Mean \pm SD & $16.80 \pm 16.92$ & $15.77 \pm 15.67$ & $19.93 \pm 20.16$ & \\
\hline & Median (Q1-Q3) & $12.73(4.70-21.15)$ & $11.32(4.70-21.49)$ & $14.94(4.68-20.30)$ & \\
\hline & Total fat (g) & & & & \multirow{3}{*}{0.08} \\
\hline & Mean $\pm S D$ & $94.17 \pm 35.33$ & $91.89 \pm 35.69$ & $101.05 \pm 33.77$ & \\
\hline & Median (Q1-Q3) & $89.87(69.68-113.81)$ & $87.51(68.86-107.90)$ & 97.99 (74.64-121.04) & \\
\hline & Total $\omega 3$ & & & & \multirow{3}{*}{0.38} \\
\hline & Mean \pm SD & $2.17 \pm 0.90$ & $2.16 \pm 0.96$ & $2.20 \pm 0.70$ & \\
\hline & Median (Q1-Q3) & $2.00(1.64-2.53)$ & $1.96(1.61-2.52)$ & $2.07(1.64-2.79)$ & \\
\hline & ALA & & & & \multirow{3}{*}{0.09} \\
\hline & Mean \pm SD & $1.76 \pm 0.73$ & $1.72 \pm 0.75$ & $1.87 \pm 0.67$ & \\
\hline & Median (Q1-Q3) & $1.64(1.30-2.12)$ & $1.58(1.28-2.02)$ & $1.84(1.35-2.23)$ & \\
\hline & LCw3 & & & & \multirow{3}{*}{0.09} \\
\hline & Mean \pm SD & $0.41 \pm 0.35$ & $0.44 \pm 0.38$ & $0.32 \pm 0.26$ & \\
\hline & Median (Q1-Q3) & $0.32(0.17-0.57)$ & $0.35(0.19-0.62)$ & $0.22(0.16-0.44)$ & \\
\hline & EPA & & & & \multirow{3}{*}{0.15} \\
\hline & Mean \pm SD & $0.15 \pm 0.15$ & $0.16 \pm 0.16$ & $0.11 \pm 0.11$ & \\
\hline & Median (Q1-Q3) & $0.11(0.05-0.19)$ & $0.12(0.05-0.20)$ & $0.08(0.05-0.16)$ & \\
\hline & DHA & & & & \multirow{3}{*}{0.08} \\
\hline & Mean \pm SD & $0.22 \pm 0.19$ & $0.24 \pm 0.20$ & $0.18 \pm 0.14$ & \\
\hline & Median (Q1-Q3) & $0.17(0.09-0.29)$ & $0.19(0.11-0.33)$ & $0.12(0.09-0.25)$ & \\
\hline & DPA & & & & \multirow{3}{*}{0.17} \\
\hline & Mean \pm SD & $0.04 \pm 0.04$ & $0.04 \pm 0.04$ & $0.03 \pm 0.03$ & \\
\hline & Median (Q1-Q3) & $0.03(0.02-0.06)$ & $0.03(0.02-0.06)$ & $0.03(0.02-0.04)$ & \\
\hline & Total $\omega 6$ & & & & \multirow{3}{*}{0.03} \\
\hline & Mean \pm SD & $14.91 \pm 6.37$ & $14.23 \pm 6.10$ & $16.96 \pm 6.80$ & \\
\hline & Median (Q1-Q3) & $13.47(10.36-18.64)$ & $13.25(10.18-17.05)$ & $17.28(10.62-22.00)$ & \\
\hline & $\omega 3 / \omega 6$ ratio & & & & \multirow{3}{*}{0.05} \\
\hline & Mean \pm SD & $0.15 \pm 0.05$ & $0.16 \pm 0.05$ & $0.14 \pm 0.04$ & \\
\hline & Median (Q1-Q3) & $0.15(0.12-0.18)$ & $0.16(0.12-0.18)$ & $0.14(0.11-0.16)$ & \\
\hline & 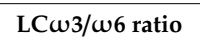 & & & & \multirow{3}{*}{0.02} \\
\hline & Mean \pm SD & $0.03 \pm 0.03$ & $0.03 \pm 0.03$ & $0.02 \pm 0.01$ & \\
\hline & Median (Q1-Q3) & $0.02(0.01-0.04)$ & $0.03(0.01-0.04)$ & $0.02(0.01-0.03)$ & \\
\hline \multirow{27}{*}{$\mathrm{RBC}^{+}$} & Total $\omega 3$ & & & & \\
\hline & Mean \pm SD & $7.98 \pm 1.42$ & $8.04 \pm 1.47$ & $7.80 \pm 1.26$ & 0.43 \\
\hline & Median (Q1-Q3) & $7.75(6.99-8.80)$ & $7.76(7.06-8.80)$ & $7.75(6.91-8.82)$ & \\
\hline & ALA & & & & \\
\hline & Mean \pm SD & $0.12 \pm 0.08$ & $0.12 \pm 0.08$ & $0.10 \pm 0.07$ & 0.30 \\
\hline & Median (Q1-Q3) & $0.14(0.00-0.17)$ & $0.14(0.00-0.17)$ & $0.14(0.00-0.16)$ & \\
\hline & LCw3 & & & & \\
\hline & Mean \pm SD & $7.79 \pm 1.42$ & $7.86 \pm 1.50$ & $7.60 \pm 1.13$ & 0.52 \\
\hline & Median (Q1-Q3) & $7.57(6.80-8.58)$ & $7.57(6.93-8.63)$ & $7.35(6.71-8.54)$ & \\
\hline & EPA & & & & \\
\hline & Mean \pm SD & $0.86 \pm 0.43$ & $0.88 \pm 0.46$ & $0.77 \pm 0.27$ & 0.19 \\
\hline & Median (Q1-Q3) & $0.79(0.60-0.98)$ & $0.81(0.60-0.99)$ & $0.72(0.61-0.89)$ & \\
\hline & DHA & & & & \\
\hline & Mean \pm SD & $4.22 \pm 0.93$ & $4.24 \pm 0.98$ & $4.13 \pm 0.79$ & 0.70 \\
\hline & Median (Q1-Q3) & 4.07 (3.59-4.75) & $4.10(3.59-4.89)$ & $4.05(3.50-4.67)$ & \\
\hline & DPA & & & & \\
\hline & Mean \pm SD & $2.72 \pm 0.38$ & $2.73 \pm 0.39$ & $2.69 \pm 0.33$ & 0.76 \\
\hline & Median (Q1-Q3) & $2.65(2.46-2.91)$ & $2.66(2.43-2.91)$ & $2.64(2.52-2.85)$ & \\
\hline & Total $\omega 6$ & & & & \\
\hline & Mean \pm SD & $28.52 \pm 1.67$ & $28.40 \pm 1.79$ & $28.90 \pm 1.18$ & 0.21 \\
\hline & Median (Q1-Q3) & $28.76(27.75-29.65)$ & $28.66(27.48-29.55)$ & $28.82(28.16-29.86)$ & \\
\hline & $\omega 3 / \omega 6$ ratio & & & & \\
\hline & Mean \pm SD & $0.28 \pm 0.07$ & $0.29 \pm 0.07$ & $0.27 \pm 0.05$ & 0.30 \\
\hline & Median (Q1-Q3) & $0.27(0.24-0.31)$ & $0.27(0.24-0.31)$ & $0.26(0.23-0.31)$ & \\
\hline & 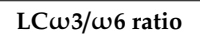 & & & & \\
\hline & Mean \pm SD & $0.27 \pm 0.07$ & $0.28 \pm 0.07$ & $0.26 \pm 0.05$ & 0.36 \\
\hline & Median (Q1-Q3) & $0.26(0.23-0.31)$ & $0.26(0.24-0.31)$ & $0.25(0.22-0.31)$ & \\
\hline
\end{tabular}


Table 2. Cont.

\begin{tabular}{|c|c|c|c|c|c|}
\hline Method & Variables & $\begin{array}{c}\text { Total } \\
n=157\end{array}$ & $\begin{array}{c}\text { Low-Grade } \\
n=118\end{array}$ & $\begin{array}{c}\text { High-Grade } \\
n=39\end{array}$ & $p$-Value $\S$ \\
\hline \multirow{27}{*}{$\begin{array}{l}\text { Prostate } \\
\text { tissue }\end{array}$} & Total $\omega 3$ & & & & \multirow{3}{*}{0.05} \\
\hline & Mean \pm SD & $3.52 \pm 1.01$ & $3.62 \pm 1.05$ & $3.23 \pm 0.81$ & \\
\hline & Median (Q1-Q3) & $3.36(2.75-4.13)$ & $3.52(2.76-4.20)$ & $3.08(2.57-3.84)$ & \\
\hline & ALA & & & & \multirow{3}{*}{0.40} \\
\hline & Mean \pm SD & $0.45 \pm 0.33$ & $0.44 \pm 0.33$ & $0.48 \pm 0.33$ & \\
\hline & Median (Q1-Q3) & $0.46(0.17-0.69)$ & $0.43(0.17-0.65)$ & $0.57(0.16-0.73)$ & \\
\hline & $\mathrm{LC} \omega 3$ & & & & \multirow{3}{*}{0.13} \\
\hline & Mean \pm SD & $2.75 \pm 1.13$ & $2.84 \pm 1.17$ & $2.50 \pm 0.98$ & \\
\hline & Median (Q1-Q3) & $2.70(1.90-3.40)$ & $2.76(1.96-3.50)$ & $2.29(1.82-3.22)$ & \\
\hline & EPA & & & & \multirow{3}{*}{0.01} \\
\hline & Mean \pm SD & $0.14 \pm 0.15$ & $0.16 \pm 0.16$ & $0.09 \pm 0.08$ & \\
\hline & Median (Q1-Q3) & $0.13(0.00-0.20)$ & $0.14(0.05-0.21)$ & $0.10(0.00-0.15)$ & \\
\hline & DHA & & & & \multirow{3}{*}{0.20} \\
\hline & Mean \pm SD & $1.76 \pm 0.80$ & $1.81 \pm 0.81$ & $1.61 \pm 0.75$ & \\
\hline & Median (Q1-Q3) & $1.75(1.10-2.35)$ & $1.83(1.10-2.39)$ & $1.44(1.03-2.21)$ & \\
\hline & DPA & & & & \multirow{3}{*}{0.20} \\
\hline & Mean \pm SD & $0.85 \pm 0.31$ & $0.87 \pm 0.32$ & $0.80 \pm 0.28$ & \\
\hline & Median (Q1-Q3) & $0.82(0.60-0.98)$ & $0.83(0.61-0.98)$ & $0.72(0.60-1.00)$ & \\
\hline & Total $\omega 6$ & & & & \multirow{3}{*}{0.95} \\
\hline & Mean \pm SD & $21.97 \pm 3.74$ & $21.98 \pm 3.70$ & $21.97 \pm 3.89$ & \\
\hline & Median (Q1-Q3) & $22.10(19.19-24.25)$ & $22.32(19.19-24.15)$ & $21.69(18.89-25.96)$ & \\
\hline & $\omega 3 / \omega 6$ ratio & & & & \multirow{3}{*}{$<0.01$} \\
\hline & Mean \pm SD & $0.16 \pm 0.04$ & $0.17 \pm 0.04$ & $0.15 \pm 0.02$ & \\
\hline & Median (Q1-Q3) & $0.16(0.14-0.17)$ & $0.16(0.14-0.18)$ & $0.14(0.13-0.16)$ & \\
\hline & 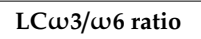 & & & & \multirow{3}{*}{0.04} \\
\hline & Mean \pm SD & $0.12 \pm 0.04$ & $0.13 \pm 0.04$ & $0.11 \pm 0.03$ & \\
\hline & Median (Q1-Q3) & $0.12(0.10-0.14)$ & $0.12(0.10-0.14)$ & $0.11(0.09-0.13)$ & \\
\hline
\end{tabular}

Abbreviations: LC $\omega 3$ = long-chain omega-3 fatty acids (EPA + DPA + DHA); EPA = Eicosapentaenoic acid; DPA $=$ Docosapentaenoic acid; DHA $=$ Docosahexaenoic acid; ALA $=$ alpha-Linolenic acid. Note: ${ }^{*}=$ Fatty acids are expressed as a daily intake (g); $\dagger=$ fatty acids are expressed as $\%$ of total fatty acids in RBC membranes; $\ddagger=$ fatty acids are expressed as $\%$ of total fatty acids in prostate tissue; $\S=p$-values were obtained using the Wilcoxon test. $\omega 3=$ omega- $3 ; \omega 6=$ omega- 6 . Bold font indicates significance at $p<0.05$.

The enzymatic activity for long-chain (LC) FA metabolism in prostate tissue was estimated for $\omega 3$ and $\omega 6$. As shown in Table 5, we found that compared to high-grade, low-grade PCa group had a higher activity of $\Delta 5$-desaturase, which converts eicosatetraenoic acid (ETA) to EPA, as well as a lower activity of Elovl2 elongase, which converts EPA to docosapentaenoic acid (DPA), altogether suggesting an EPA bioaccumulation in prostate tissue of low-grade PCa (Supplementary Figure S2).

Associations between FA levels in RBC and reclassification to high-grade PCa are presented in Table 6. We observed a similar direction of effect of the LC $\omega 3 / \omega 6$ ratio measured in RBC than that measured in prostate tissue, but none of the RBC associations were significant (all $p$-trends $>0.20$ ).

The associations between dietary FA intake and reclassification to high-grade PCa are presented in Table 7. Contrasting with FA levels in RBC, men in the highest tertile of LC $\omega 3$ intake had a decreased risk of high-grade PCa (adjusted OR 0.30; 95\% CI 0.11-0.83; $p$-trend $=0.02$ ) compared to men in the lowest tertile of LCw3 intake. A similar protective effect was observed with a higher LCw3/w6 ratio $($ OR $0.29 ; 95 \%$ CI $0.11-0.76$; $p$-trend $=0.03$ ). 
Table 3. Associations between fatty acid profiles of prostate tissue and high-grade prostate cancer.

\begin{tabular}{|c|c|c|c|c|c|c|c|}
\hline \multirow{3}{*}{$\begin{array}{c}\text { Fatty Acid } \\
\text { w3 total }\end{array}$} & \multirow{3}{*}{$\begin{array}{c}\text { Tertile } \\
1\end{array}$} & \multicolumn{3}{|c|}{ Fatty Acids (\%) * } & \multicolumn{3}{|c|}{ Fatty Acids (mg/g) † } \\
\hline & & \multirow{2}{*}{$\begin{array}{c}n \text { High-Grade/ } \\
\boldsymbol{n} \text { Low-Grade }\end{array}$} & \multicolumn{2}{|c|}{$\begin{array}{l}\text { Multivariable Models } \ddagger \\
\text { OR }(95 \% \text { CI) } p \text {-Value } §\end{array}$} & \multirow{2}{*}{$\begin{array}{c}n \text { High-Grade/ } \\
\boldsymbol{n} \text { Low-Grade }\end{array}$} & \multicolumn{2}{|c|}{$\begin{array}{l}\text { Multivariable Models } \\
\text { OR }(95 \% \text { CI }) p \text {-Value } \S\end{array}$} \\
\hline & & & 1 & & & 1 & \\
\hline & 2 & $16 / 37$ & $1.24(0.50-3.07)$ & 0.64 & $10 / 40$ & $0.68(0.26-1.81)$ & 0.44 \\
\hline & 3 & $8 / 43$ & $0.49(0.18-1.36)$ & 0.17 & $13 / 39$ & $0.79(0.31-2.02)$ & 0.63 \\
\hline & & & p-trend $\|$ & 0.15 & & & 0.68 \\
\hline \multirow[t]{4}{*}{ ALA } & 1 & $14 / 37$ & 1 & & $13 / 40$ & 1 & \\
\hline & 2 & $8 / 46$ & 0.39 (0.14-1.12) & 0.08 & $11 / 41$ & $0.92(0.35-2.41)$ & 0.86 \\
\hline & 3 & $17 / 35$ & $1.35(0.54-3.42)$ & 0.51 & $15 / 37$ & $1.21(0.48-3.07)$ & 0.68 \\
\hline & & & $p$-trend $\|$ & 0.68 & & & 0.65 \\
\hline \multirow[t]{4}{*}{ LCw3 } & 1 & $17 / 37$ & 1 & & $14 / 37$ & 1 & \\
\hline & 2 & $11 / 40$ & $0.68(0.26-1.78)$ & 0.44 & $16 / 39$ & $1.03(0.41-2.57)$ & 0.95 \\
\hline & 3 & $11 / 41$ & $0.59(0.23-1.51)$ & 0.27 & $9 / 42$ & $0.59(0.21-1.65)$ & 0.31 \\
\hline & & & $p$-trend $\|$ & 0.27 & & & 0.28 \\
\hline \multirow[t]{4}{*}{ EPA } & 1 & $16 / 37$ & 1 & & $15 / 31$ & 1 & \\
\hline & 2 & $18 / 36$ & $1.35(0.55-3.31)$ & 0.50 & $14 / 28$ & $1.02(0.39-2.69)$ & 0.95 \\
\hline & 3 & $5 / 45$ & $0.25(0.08-0.79)$ & 0.02 & $10 / 59$ & $0.35(0.13-0.92)$ & 0.03 \\
\hline & & & p-trend $\|$ & 0.03 & & & 0.03 \\
\hline \multirow[t]{4}{*}{ DHA } & 1 & $16 / 37$ & 1 & & $13 / 39$ & 1 & \\
\hline & 2 & $11 / 41$ & $0.78(0.29-2.08)$ & 0.62 & $16 / 38$ & $1.43(0.57-3.62)$ & 0.45 \\
\hline & 3 & $12 / 40$ & $0.74(0.29-1.90)$ & 0.54 & $10 / 41$ & $0.74(0.27-2.10)$ & 0.58 \\
\hline & & & $p$-trend $\|$ & 0.54 & & & 0.55 \\
\hline \multirow[t]{4}{*}{ DPA } & 1 & $16 / 36$ & 1 & & $17 / 34$ & 1 & \\
\hline & 2 & $12 / 40$ & $0.73(0.29-1.86)$ & 0.24 & $11 / 40$ & $0.57(0.22-1.48)$ & 0.25 \\
\hline & 3 & $11 / 42$ & $0.59(0.23-1.53)$ & 0.28 & $11 / 44$ & $0.50(0.19-1.27)$ & 0.14 \\
\hline & & & $p$-trend $\|$ & 0.28 & & & 0.17 \\
\hline \multirow[t]{4}{*}{$\omega 6$ total } & 1 & $13 / 40$ & 1 & & $16 / 37$ & 1 & \\
\hline & 2 & $12 / 38$ & $1.35(0.49-3.74)$ & 0.55 & $6 / 46$ & $0.32(0.11-0.96)$ & 0.04 \\
\hline & 3 & $14 / 40$ & $1.28(0.48-3.40)$ & 0.62 & $17 / 35$ & $1.14(0.47-2.77)$ & 0.77 \\
\hline & & & $p$-trend $\|$ & 0.64 & & & 0.54 \\
\hline Ratio & 1 & $19 / 34$ & 1 & & $19 / 34$ & 1 & \\
\hline \multirow[t]{3}{*}{$\omega 3 / \omega 6$} & 2 & $14 / 39$ & $0.55(0.22-1.34)$ & 0.19 & $13 / 40$ & $0.50(0.20-1.24)$ & 0.13 \\
\hline & 3 & $6 / 45$ & $0.20(0.07-0.59)$ & $<0.01$ & $7 / 44$ & $0.24(0.09-0.68)$ & $<0.01$ \\
\hline & & & $p$-trend $\|$ & $<0.01$ & & & $<0.01$ \\
\hline Ratio & 1 & $17 / 36$ & 1 & & $17 / 36$ & 1 & \\
\hline \multirow[t]{3}{*}{$\mathrm{LC} \omega 3 / \omega 6$} & 2 & $13 / 39$ & $0.73(0.29-1.86)$ & 0.51 & $13 / 39$ & $0.79(0.31-2.00)$ & 0.62 \\
\hline & 3 & $9 / 43$ & $0.38(0.14-1.00)$ & 0.05 & $9 / 43$ & $0.42(0.16-1.10)$ & 0.07 \\
\hline & & & $p$-trend $\|$ & 0.05 & & & 0.07 \\
\hline
\end{tabular}

NOTE: Logistic regression models in which outcome is the presence of high-risk prostate cancer defined as grade group $\geq 2$ at the first repeat biopsy session versus low-risk prostate cancer. Note: ${ }^{*}=$ Prostatic fatty acids were expressed as $\%$ of total fatty acids of prostatic biopsy; $+=$ prostatic fatty acids were expressed in absolute concentrations (mg of fatty acid per $\mathrm{g}$ of prostatic biopsy); $\ddagger=$ multivariable models were adjusted for age, prostate-specific antigen (PSA) level, waist circumference, physical activity, alcohol, and total energy intake; $\S=$ p-value of category relative to referent (lowest tertile); $\|=$ adjusted trend $p$-value. Abbreviations: EPA = Eicosapentaenoic acid; DPA = Docosapentaenoic acid; DHA = Docosahexaenoic acid; ALA = alpha-Linolenic acid; Long-chain $\omega 3$ (LCw3) = EPA + DHA + DPA. OR = odds ratio; CI = confidence interval. Bold font indicates significance at $p<0.05$.

Table 4. Trend analysis of EPA level in prostate tissue.

\begin{tabular}{cccccc}
\hline \multirow{2}{*}{ EPA Level * } & $\boldsymbol{n}$ & \multicolumn{2}{c}{ Frequency, $\mathbf{n}(\%)$} & \multirow{2}{*}{$\boldsymbol{p}$-Trend ${ }^{\dagger}$} & \multirow{2}{*}{ Adjusted $\boldsymbol{p}$-Trend $\ddagger$} \\
\cline { 3 - 4 } & & Low-Grade & High-Grade & & \\
\hline 0 & 44 & $29(66 \%)$ & $15(34 \%)$ & $<\mathbf{0 . 0 1}$ & $\mathbf{0 . 0 1}$ \\
$0-0.2$ & 73 & $52(71 \%)$ & $21(29 \%)$ & & \\
$0.2-0.4$ & 33 & $30(91 \%)$ & $3(9 \%)$ & & \\
$\geq 0.4$ & 7 & $7(100 \%)$ & $0(0 \%)$ & & \\
\hline
\end{tabular}

Note: ${ }^{*}=$ EPA level is expressed as $\%$ of EPA on total FA of prostate tissue; $\uparrow=p$-trend was calculated using Mantel Haenszel test; $\ddagger=$ Adjusted $p$-trend was obtained by treating the median of each category as a continuous variable in multivariable regression model adjusted for age, PSA level, waist circumference, physical activity, alcohol, and total energy intake. The two last categories were merged together to bypass the complete separation issue for logistic regression. Bold font indicates significance at $p<0.05$. 
Table 5. Estimation of elongase and desaturase activity in high- and low-grade PCa groups.

\begin{tabular}{|c|c|c|c|c|c|}
\hline \multirow{2}{*}{$\begin{array}{c}\text { Fatty Acid } \\
\text { Ratio }\end{array}$} & \multirow{2}{*}{$\begin{array}{c}\text { Fatty Acid } \\
\text { Ratio }\end{array}$} & \multirow{2}{*}{$\begin{array}{c}\text { Enzymatic } \\
\text { Activity }\end{array}$} & \multicolumn{2}{|c|}{ mean $\pm S D$} & \multirow{2}{*}{$p$-Value * } \\
\hline & & & High-Grade & Low-Grade & \\
\hline $20: 3 n 6 / 18: 3 n 6$ & DGLA/GLA & Elovl5 elongase & $14.07 \pm 6.06$ & $15.27 \pm 5.14$ & 0.42 \\
\hline $22: 4 n 6 / 20: 4 n 6$ & Adrenic/AA & Elovl2 elongase & $0.16 \pm 0.03$ & $0.15 \pm 0.04$ & 0.13 \\
\hline $20: 4 n 3 / 18: 4 n 3$ & ETA/Stearidonic & Elovl5 elongase & $0.61 \pm 0.35$ & $0.76 \pm 0.50$ & 0.77 \\
\hline $22: 5 n 3 / 20: 5 n 3$ & DPA/EPA & Elovl2 elongase & $6.00 \pm 1.68$ & $5.32 \pm 2.14$ & 0.01 \\
\hline 18:1n9/18:0 & Oleic/Stearic & $\Delta 9$-desaturase & $3.22 \pm 1.96$ & $2.90 \pm 1.86$ & 0.42 \\
\hline $18: 3 \mathrm{n} 6 / 18: 2 \mathrm{n} 6$ & GLA/LA & $\Delta 6$-desaturase & $0.01 \pm 0.01$ & $0.01 \pm 0.01$ & 0.50 \\
\hline $18: 4 n 3 / 18: 3 n 3$ & Stearidonic/ALA & $\Delta 6$-desaturase & $0.82 \pm 0.81$ & $0.84 \pm 0.88$ & 0.58 \\
\hline $20: 4 n 6 / 20: 3 n 6$ & AA/DGLA & $\Delta 5$-desaturase & $9.80 \pm 21.21$ & $4.86 \pm 1.17$ & 0.36 \\
\hline $20: 5 n 3 / 20: 4 n 3$ & EPA/ETA & $\Delta 5$-desaturase & $1.32 \pm 0.73$ & $1.72 \pm 1.07$ & 0.02 \\
\hline
\end{tabular}

Abbreviations: DGLA = Dihomo-gamma linolenic acid dihomo-gamma; GLA = gamma linolenic acid; $\mathrm{ETA}=$ Eicosatetraenoic acid; DPA = Docosapentaenoic acid; ALA = Alpha-linolenic acid; $\mathrm{AA}=$ Arachidonic acid; $\mathrm{LA}=$ Linoleic acid. Bold font indicates significance at $p<0.05 .{ }^{*}=p$-values were obtained using the Wilcoxon test.

Table 6. Associations between fatty acid profiles of RBC and high-grade prostate cancer.

\begin{tabular}{|c|c|c|c|c|c|}
\hline \multirow{2}{*}{ Fatty Acid } & \multirow{2}{*}{ Tertile } & \multirow{2}{*}{$\begin{array}{c}n \text { High-Grade/ } \\
n \text { Low-Grade }\end{array}$} & \multicolumn{3}{|c|}{ Multivariable Models* } \\
\hline & & & OR $(95 \%$ CI) & $p$-Value ${ }^{\dagger}$ & $p$-Trend $\ddagger$ \\
\hline \multirow[t]{3}{*}{$\omega 3$ total } & 1 & $17 / 36$ & 1 & & 0.24 \\
\hline & 2 & $11 / 42$ & $0.52(0.20-1.31)$ & 0.16 & \\
\hline & 3 & $11 / 40$ & $0.58(0.23-1.48)$ & 0.25 & \\
\hline \multirow[t]{3}{*}{ ALA } & 1 & $14 / 37$ & 1 & & 0.68 \\
\hline & 2 & $17 / 43$ & $1.23(0.50-3.00)$ & 0.65 & \\
\hline & 3 & $8 / 38$ & $0.60(0.23-1.83)$ & 0.42 & \\
\hline \multirow[t]{3}{*}{$\mathrm{LC} \omega 3$} & 1 & $15 / 37$ & 1 & & 0.43 \\
\hline & 2 & $13 / 41$ & $0.70(0.27-1.76)$ & 0.44 & \\
\hline & 3 & $11 / 40$ & $0.68(0.26-1.76)$ & 0.42 & \\
\hline \multirow{3}{*}{ EPA } & 1 & $12 / 39$ & 1 & & 0.33 \\
\hline & 2 & $18 / 37$ & $1.46(0.58-3.65)$ & 0.42 & \\
\hline & 3 & $9 / 42$ & $0.61(0.21-1.73)$ & 0.35 & \\
\hline \multirow[t]{3}{*}{ DHA } & 1 & $13 / 40$ & 1 & & 0.50 \\
\hline & 2 & $16 / 37$ & $1.11(0.44-2.81)$ & 0.82 & \\
\hline & 3 & $10 / 41$ & $0.73(0.27-1.99)$ & 0.54 & \\
\hline \multirow[t]{3}{*}{ DPA } & 1 & $10 / 43$ & 1 & & 0.93 \\
\hline & 2 & $19 / 33$ & $2.85(1.06-7.70)$ & 0.04 & \\
\hline & 3 & $10 / 42$ & $1.18(0.42-3.36)$ & 0.75 & \\
\hline \multirow[t]{3}{*}{$\omega 6$ total } & 1 & $9 / 43$ & 1 & & 0.20 \\
\hline & 2 & $15 / 37$ & $1.95(0.72-5.32)$ & 0.18 & \\
\hline & 3 & $15 / 38$ & $1.92(0.70-5.26)$ & 0.20 & \\
\hline \multirow[t]{3}{*}{ Ratio $\omega 3 / \omega 6$} & 1 & $16 / 37$ & 1 & & 0.23 \\
\hline & 2 & $13 / 40$ & $0.70(0.28-1.76)$ & 0.45 & \\
\hline & 3 & $10 / 41$ & $0.55(0.21-1.45)$ & 0.22 & \\
\hline \multirow[t]{3}{*}{ Ratio LC $\omega 3 / \omega-6$} & 1 & $15 / 38$ & 1 & & 0.24 \\
\hline & 2 & $14 / 39$ & $0.80(0.32-2.00)$ & 0.64 & \\
\hline & 3 & $10 / 41$ & $0.56(0.21-1.49)$ & 0.25 & \\
\hline
\end{tabular}

Note: Logistic regression models in which outcome is the presence of high-risk prostate cancer defined as grade group $\geq 2$ at the first repeat biopsy session versus low-risk prostate cancer; ${ }^{*}=$ Multivariable models were adjusted for age, PSA level, waist circumference, physical activity, alcohol, and total energy intake; $+=p$-value of category relative to referent (lowest tertile); $\ddagger=$ adjusted trend $p$-value; $E P A=$ Eicosapentaenoic acid; DPA $=$ Docosapentaenoic acid; DHA = Docosahexaenoic acid; ALA = alpha-Linolenic acid; Long-chain $\omega 3$ (LC $\omega 3$ ) = EPA + DHA + DPA. 
Table 7. Associations between fatty acid intake assessed by the web-FFQ and high-grade prostate cancer.

\begin{tabular}{|c|c|c|c|c|c|}
\hline \multirow{2}{*}{ Fatty Acid } & \multirow{2}{*}{ Tertile } & \multirow{2}{*}{$\begin{array}{c}n \text { High-Grade/ } \\
n \text { Low-Grade }\end{array}$} & \multicolumn{3}{|c|}{ Multivariable Models * } \\
\hline & & & OR $(95 \%$ CI) & $p$-Value ${ }^{\dagger}$ & $p$-Trend \\
\hline \multirow[t]{3}{*}{$\omega 3$ total } & 1 & $11 / 43$ & 1 & & 0.75 \\
\hline & 2 & $15 / 39$ & $1.69(0.61-4.70)$ & 0.31 & \\
\hline & 3 & $13 / 36$ & $1.40(0.39-5.02)$ & 0.60 & \\
\hline \multirow{3}{*}{ ALA } & 1 & $12 / 42$ & 1 & & 0.17 \\
\hline & 2 & $10 / 43$ & $0.99(0.32-3.05)$ & 0.99 & \\
\hline & 3 & $17 / 33$ & $2.29(0.58-9.07)$ & 0.24 & \\
\hline \multirow[t]{3}{*}{$\mathrm{LC} \omega 3$} & 1 & $19 / 34$ & 1 & & 0.02 \\
\hline & 2 & $11 / 43$ & $0.38(0.15-0.98)$ & 0.04 & \\
\hline & 3 & $9 / 41$ & $0.30(0.11-0.83)$ & 0.02 & \\
\hline \multirow[t]{3}{*}{ EPA } & 1 & $16 / 37$ & 1 & & 0.13 \\
\hline & 2 & $13 / 41$ & $0.63(0.25-1.60)$ & 0.33 & \\
\hline & 3 & $10 / 40$ & $0.46(0.17-1.26)$ & 0.13 & \\
\hline \multirow{3}{*}{ DHA } & 1 & $18 / 35$ & 1 & & 0.02 \\
\hline & 2 & $13 / 41$ & $0.52(0.20-1.30)$ & 0.16 & \\
\hline & 3 & $8 / 42$ & $0.29(0.10-0.83)$ & 0.02 & \\
\hline \multirow[t]{3}{*}{ DPA } & 1 & $14 / 39$ & 1 & & 0.12 \\
\hline & 2 & $17 / 37$ & $1.05(0.43-2.58)$ & 0.90 & \\
\hline & 3 & $8 / 42$ & 0.47 (0.17-1.32) & 0.15 & \\
\hline \multirow[t]{3}{*}{$\omega 6$ total } & 1 & $13 / 40$ & 1 & & 0.04 \\
\hline & 2 & $5 / 46$ & $0.33(0.10-1.13)$ & 0.08 & \\
\hline & 3 & $21 / 32$ & $2.22(0.64-7.78)$ & 0.20 & \\
\hline \multirow[t]{3}{*}{ Ratio $\omega 3 / \omega 6$} & 1 & $17 / 36$ & 1 & & 0.17 \\
\hline & 2 & $13 / 40$ & $0.75(0.31-1.87)$ & 0.55 & \\
\hline & 3 & $9 / 42$ & $0.50(0.19-1.36)$ & 0.18 & \\
\hline \multirow[t]{3}{*}{ Ratio LC $\omega 3 / \omega 6$} & 1 & $21 / 32$ & 1 & & 0.03 \\
\hline & 2 & $9 / 43$ & $0.27(0.10-0.71)$ & $<0.01$ & \\
\hline & 3 & $9 / 43$ & $0.29(0.11-0.76)$ & 0.01 & \\
\hline
\end{tabular}

Note: Logistic regression models in which outcome is the presence of high-risk prostate cancer defined as grade group $\geq 2$ at the first repeat biopsy session versus low-risk prostate cancer; ${ }^{*}=$ Multivariable models were adjusted for age, PSA level, waist circumference, physical activity, alcohol. and total energy intake; $t=p$-value of category relative to referent (lowest tertile); $\ddagger=$ adjusted trend p-value; EPA = Eicosapentaenoic acid; DPA = Docosapentaenoic acid; DHA = Docosahexaenoic acid; ALA = alpha-Linolenic acid; Long-chain $\omega 3$ (LC $\omega 3)=$ EPA + DHA + DPA. Bold font indicates significance at $p<0.05$.

\section{Discussion}

This is a validation study of the first and only study that evaluated the associations between high-grade PCa and FA content measured in the prostate tissue during active surveillance, moreover linking it to RBC and diet. The opportunity of an ongoing randomized controlled trial in men starting active surveillance was ideally suited to examine that question. We found that high level of EPA, a subtype of LC $\omega 3$, was associated with a decreased risk of high-grade disease in the prostate tissue, and found a significant dose-response association ( $p$-trend $<0.01$ ). We also observed that higher $\omega 3 / \omega 6$ and LC $\omega 3 / \omega 6$ ratios decreased risk of reclassification to high-grade disease. This validation study supports our a priori hypothesis, based on our initial study [28], that higher level of $\omega 3$, particularly EPA, is inversely associated with risk of high-grade PCa. This current study represents an important opportunity to re-examine this question under the lens of information bias, given that two recent studies reported conflicting results about the role of $\omega 3$ on PCa $[18,24]$. These observational studies were conducted within large-scale randomized trials testing other interventions on PCa incidence. Such studies are subject to prognostic selection bias, potentially limiting the generalizability of study findings.

More importantly, the prostate gland is metabolically active. Indeed, the estimated prostatic FA metabolism was also linked to high-grade PCa. Compared to the high-grade group, the low-grade group had a higher activity of $\triangle 5$-desaturase, which converts ETA to EPA, while the activity of Elovl2 elongase, which converts EPA to DPA, was significantly lower. Thus, the prostatic FA metabolism, 
which translates to elevated EPA levels, decreased the risk of high-grade disease. This supports our hypothesis that EPA may be a key protector against PCa aggressiveness.

In order to evaluate if the FA measurement in RBC can be used as biomarker for the prostate FA content, and hence a PCa biomarker, we also examined its relation with PCa grade. We observed that none of the FA measured in RBC were significantly associated with PCa grade. Several methodologic distinctions of our study are worth mentioning to support our findings. Our bio-banking protocol included drawing blood in the early morning after overnight fasting, as well as treatment of bio-specimens within one hour of collection to prevent FA oxidation, which affects the accuracy of FA profiles. These procedures were not part of any of the previous blood FA biomarker studies. Moreover, FA profiles measured in RBC are much less affected by the recent dietary intake and circadian variation than is the FA profile measure in plasma phospholipids, which was used in the vast majority of LC $\omega 3$ biomarkers studies of PCa risk, including the two large-scale recent ones [18,24]. The RBC FA profile rather reflects the FA content of diet of the last three months. One explanation for the lack of association between blood biomarker and PCa grade might also be that the RBCs, being anucleated, reflect both dietary intake and FA metabolism of the whole body, not that specifically of the prostate. In fact, we observed a regression to the mean effect of the same direction of associations with loss of significance. In addition, the FA level correlations between RBC and prostate tissue were weak, at best moderate (Supplementary Table S2). Unfortunately, RBC FA profile does not seem to be a good biomarker for PCa compared to FA profile in the prostatic tissue.

Since LC $\omega 3$ FA are essential, we also verified the association with $\omega 3$ measured in the diet. We observed, in line with the prostate content, that a high dietary content in LC $\omega 3$ and LC $\omega 3 / \omega 6$ were associated with a deceased risk of high-grade disease. This contrasts with the absence of association with FA levels in RBC. This suggests that there may be other protector elements in the diet in addition to LC $w 3$ alone. Interestingly, while we found no such data in cancer patients, we found a trial in insulin-resistant patients that have compared the source of $\omega 3$. Patients consumed equal amounts of LC $\omega 3$ directly from fish versus from processed fish oil. Only fish-consumed LC $\omega 3$ reduced C-reactive protein, a marker of systemic inflammation, and improved insulin sensitivity [38,39]. Thus, the dietary source of LCw3 may matter more for clinical inflammatory-related effects than their blood levels alone. In our current study, even though small sample size precluded most post-hoc subgroup analyses, we observed that greater fish intake (at least once a week) decreased the risk of high-grade PCa (adjusted OR 0.40; 95\% CI 0.16-0.98; $p=0.04$ ), while intake of plant sources of LC $\omega 3$, such as flaxseed and other nuts, did not (OR $0.73 ; 95 \%$ CI $0.31-1.72 ; p=0.47$ ). However, we did not find any association with vitamin $\mathrm{D}$ and other protein sources (data not shown).

Our findings of a protective association between LC $\omega 3$ and high-grade PCa are in line with previous experimental studies. In fact, most preclinical experimental studies in mice showed that a LC $\omega 3$ enriched diet reduces PCa progression [40-44]. Also, all clinical trials testing the impact of LC $\omega 3$ on PCa cell proliferation showed a significant reduction [10,11,45]. However, results from observational studies have been inconsistent. Among 30 cohorts and case-control studies investigating incidence of PCa and FA intake or biomarkers thereof, 11 reported inverse associations, similar to our study; the others showed positive or no association. These inconsistent observations may arise from various important methodological issues. First, various techniques and sample types are used to measure FA. For example, the contradictory studies have measured FA concentration in plasma or serum. Plasma levels of $\omega 3$ FA are considered a poor biomarker of long-term $\omega 3$ intake, because this measurement may be highly influenced by the last meal's content and plasma triglyceride levels, especially if patients were not fasting prior to blood collection, which was the case in the two larger recent studies $[18,24]$. These methods can lead to information bias of the exposure measure. Second, also in contrast to our study, most studies did not adjust for many potential confounding factors, such as physical activity, alcohol, and anthropometric measures. All of these may affect systemic and possibly prostate tissue inflammation, a potential causal mechanism of $\omega 3$, and other biological 
pathways leading to PCa progression [37]. Moreover, none of these studies measured the FA profile of the target prostate tissue, supporting the relevance of our study design.

To our knowledge, this is the first study to examine the relation between the FA profile of prostate tissue and high-grade PCa during active surveillance. We found only one other group who examined how prostate tissue FA profile was related to a risk measure of PCa aggressiveness [46]. In radical prostatectomy specimens, prostate tissue levels of LC $\omega 3$ (EPA + DHA) were inversely associated with the risk of locally advanced disease (OR $0.52,95 \%$ CI, $0.30-0.90 ; p=0.02$ ) [46]. There is currently no non-invasive method to quantify any tissue FA profile, and we are the first group to examine this profile while the prostate is still at risk of cancer progression. With emerging technologies, the concept of measuring metabolic aspects of prostate tissue non-invasively is becoming a reality. For example, magnetic resonance spectroscopy provides a non-invasive method of detecting small molecular biomarkers (choline-containing metabolites, polyamines, and citrate) within the prostate [47,48]. This improves diagnosis and staging of PCa $[47,48]$, and algorithms are being derived to identify prostate tissue inflammation [49-51].

This prostate tissue chronic inflammation is an increasingly supported causal mechanism of PCa development and progression [52,53]. While $\omega 6$ are pro-inflammatory, LC $\omega 3$ are anti-inflammatory, mainly because of their derived metabolites [54]. These include eicosanoids, which also have anti-neoplastic properties when deriving from LCw3 [55]. A case-control study in the placebo arm of the Prostate Cancer Prevention Trial (PCPT) observed that, as expected from studies of systemic markers of inflammation, plasma $\omega 3$ were inversely associated to prostate tissue inflammation [56]. This observation supports the findings of our current study. However, another study by Brasky et al. observed in the same PCPT study [18] found positive associations between plasma w3 FA levels and risk of PCa diagnosis. Thus, because of conflicting observational evidence, PCa risk cannot clearly be related to blood markers of LCw3 intake.

Some other methodological aspects of our study are worth mentioning. We used a cross sectional design, nested in a randomized trial, in which many methodological aspects were designed to evaluate the role of dietary FA on PCa grade. First, dietary intake was measured before the outcome ascertained, reducing possibility of recall bias, which affects some case-control studies of dietary factors. Second, the widely used and well-validated web-FFQ used in the present study measures various food preparation aspects, not measured in many FFQ, but important in FA biology, such as type of oil dressing and cooking modes. This is a great opportunity to evaluate the impact of FA on PCa. Third, rather than a risk of diagnosis model where causal factors may act during a very long period, we used an active surveillance model. This model provides an opportunity to examine the last factors leading to high-grade, potentially lethal, PCa. Inflammation could play an important role in that transition. However, some data provide uncertainty to this chain of continuity [57,58], suggesting that low-grade and higher-grade tumor nodules are different entities. Finally, in this current study, the same specialized ultrasonographist performed all study imaging procedures and tissue sampling, using a specific protocol uniform throughout the study. This eliminates inter-observer variation and contributes to increase internal validity.

In summary, this study validates in a larger cohort our previous findings suggesting that LCw3, the EPA subtype in particular, are protective against high-grade PCa. Specifically, the prostate tissue level of EPA and the LC $\omega 3 / \omega 6$ ratio were inversely associated with high-grade PCa at initiation of active surveillance in men with low-risk PCa. The dietary intake of LCw3, essential FA, and the LC $\omega 3 / \omega 6$ ratio showed similarly protective associations. In contrast, we observed both an absence of associations between grade and the RBC FA profile, and weak correlations between RBC and prostate tissue FA profiles, suggesting that blood FA biomarkers may be less useful in their capacity to predict PCa aggressiveness. This study provides a rationale for future PCa prevention studies to increase LC $\omega 3$ intake from food rather than from processed supplement sources. More studies are needed and justified to decipher the effects of LC $\omega 3$ on PCa, as well as to identify the best FA biomarker of PCa risk and aggressiveness. 
Supplementary Materials: The following are available online at http://www.mdpi.com/2072-6643/11/7/1616/s1. Table S1: Characteristics of excluded and included participants. Table S2: Correlations between fatty acid intake, fatty acid profiles of RBC membranes, and of prostate tissue. Figure S1: Distribution of eicosapentaenoic acid (EPA) level in prostate tissue. Figure S2: Schematic representation of long-chain polyunsaturated omega-6 and omega-3 fatty acids metabolism.

Author Contributions: Guarantor of integrity of the entire study, V.F.; study concept and design, V.F., P.J., B.L., B.T., and C.D.; literature research, H.M., K.R., and V.F.; clinical studies, V.F., J.A., and X.M.; experimental studies and data analysis (data acquisition), H.M., X.M., K.R., J.A., J.-F.P., N.G., B.L., P.J., B.T., C.D., P.D., and V.F; statistical analysis, H.M., M.N.-M., M.-H.G., and V.F.; manuscript preparation, H.M., M.-H.G., K.R., and V.F.; manuscript editing, M.N.-M., J.A., B.L., P.J., C.D., J.-F.P., B.T., P.D., X.M., and N.G.

Funding: This work was supported by Fonds de recherche du Québec en santé (FRQS; Researcher Award, career grants Junior 1 and Junior 2); Canadian Urological Association (CUA Foundation Scholarship Grant); American Urological Association (Young Investigator Research Grant); Prostate Cancer Canada (Clinician Scientist Award and Grant); and the Fondation du Centre Hospitalier Universitaire (CHU) de Québec.

Acknowledgments: The authors thank the participants of this study, who have contributed to a better understanding of prostate cancer dietary risk factors. They thank Claire Ménard and Hélène Hovington for their expertise, particularly helpful in the bio-specimen handling and processing, and data management. They also thank Line Berthiaume and Karine Greffard for the analysis of FA profiles.

Conflicts of Interest: The authors declare no conflict of interest.

\section{References}

1. Parkin, D.M.; Whelan, S.L.; Ferlay, J.; Teppo, L.; Thomas, D. Cancer Incidence in Five Continents, International Agency for Research on Cancer; IARC Scientific Publications: Lyon, France, 2003; Volume 155.

2. Haenszel, W.; Kurihara, M. Studies of Japanese migrants. I. Mortality from cancer and other diseases among Japanese in the United States. J. Natl. Cancer Inst. 1968, 40, 43-68. [PubMed]

3. Wilson, K.M.; Giovannucci, E.L.; Mucci, L.A. Lifestyle and dietary factors in the prevention of lethal prostate cancer. Asian J. Androl. 2012, 14, 365-374. [CrossRef] [PubMed]

4. Maskarinec, G.; Noh, J.J. The effect of migration on cancer incidence among Japanese in Hawaii. Ethn. Dis. 2004, 14, 431-439. [PubMed]

5. Carriere, G.M.; Tjepkema, M.; Pennock, J.; Goedhuis, N. Cancer patterns in Inuit Nunangat: 1998-2007. Int. J. Circumpolar Health 2012, 71, 18581. [CrossRef] [PubMed]

6. Dewailly, E.; Mulvad, G.; Sloth Pedersen, H.; Hansen, J.C.; Behrendt, N.; Hart Hansen, J.P. Inuit are protected against prostate cancer. Cancer Epidemiol. Biomarkers Prev. 2003, 12, 926-927. [PubMed]

7. Wendel, M.; Heller, A.R. Anticancer actions of omega-3 fatty acids-current state and future perspectives. Anticancer Agents Med. Chem. 2009, 9, 457-470. [CrossRef] [PubMed]

8. Tamma, S.M.; Shorter, B.; Toh, K.L.; Moldwin, R.; Gordon, B. Influence of polyunsaturated fatty acids on urologic inflammation. Int. Urol. Nephrol. 2015, 47, 1753-1761. [CrossRef] [PubMed]

9. Rose, D.P. Dietary fatty acids and prevention of hormone-responsive cancer. Proc. Soc. Exp. Biol. Med. 1997, 216, 224-233. [CrossRef]

10. Aronson, W.J.; Kobayashi, N.; Barnard, R.J.; Henning, S.; Huang, M.; Jardack, P.M.; Liu, B.; Gray, A.; Wan, J.; Konijeti, R.; et al. Phase II prospective randomized trial of a low-fat diet with fish oil supplementation in men undergoing radical prostatectomy. Cancer Prev. Res. 2011, 4, 2062-2071. [CrossRef]

11. Galet, C.; Gollapudi, K.; Stepanian, S.; Byrd, J.B.; Henning, S.M.; Grogan, T.; Elashoff, D.; Heber, D.; Said, J.; et al. Effect of a low-fat fish oil diet on proinflammatory eicosanoids and cell-cycle progression score in men undergoing radical prostatectomy. Cancer Prev. Res. 2014, 7, 97-104. [CrossRef]

12. Terry, P.; Lichtenstein, P.; Feychting, M.; Ahlbom, A.; Wolk, A. Fatty fish consumption and risk of prostate cancer. Lancet 2001, 357, 1764-1766. [CrossRef]

13. Augustsson, K.; Michaud, D.S.; Rimm, E.B.; Leitzmann, M.F.; Stampfer, M.J.; Willett, W.C.; Giovannucci, E. A prospective study of intake of fish and marine fatty acids and prostate cancer. Cancer Epidemiol. Biomarkers Prev. 2003, 12, 64-67. [PubMed]

14. Leitzmann, M.F.; Stampfer, M.J.; Michaud, D.S.; Augustsson, K.; Colditz, G.C.; Willett, W.C.; Giovannucci, E.L. Dietary intake of n-3 and n-6 fatty acids and the risk of prostate cancer. Am. J. Clin. Nutr. 2004, 80, 204-216. [CrossRef] [PubMed] 
15. Williams, C.D.; Whitley, B.M.; Hoyo, C.; Grant, D.J.; Iraggi, J.D.; Newman, K.A.; Gerber, L.; Taylor, L.A.; McKeever, M.G.; Freedland, S.J. A high ratio of dietary n-6/n-3 polyunsaturated fatty acids is associated with increased risk of prostate cancer. Nutr. Res. 2011, 31, 1-8. [CrossRef] [PubMed]

16. Norrish, A.E.; Skeaff, C.M.; Arribas, G.L.; Sharpe, S.J.; Jackson, R.T. Prostate cancer risk and consumption of fish oils: A dietary biomarker-based case-control study. Br. J. Cancer 1999, 81, 1238-1242. [CrossRef] [PubMed]

17. Terry, P.D.; Rohan, T.E.; Wolk, A. Intakes of fish and marine fatty acids and the risks of cancers of the breast and prostate and of other hormone-related cancers: A review of the epidemiologic evidence. Am. J. Clin. Nutr. 2003, 77, 532-543. [CrossRef] [PubMed]

18. Brasky, T.M.; Till, C.; White, E.; Neuhouser, M.L.; Song, X.; Goodman, P.; Thompson, I.M.; King, I.B.; Albanes, D.; Kristal, A.R. Serum phospholipid fatty acids and prostate cancer risk: Results from the prostate cancer prevention trial. Am. J. Epidemiol. 2011, 173, 1429-1439. [CrossRef] [PubMed]

19. Sala-Vila, A.; Calder, P.C. Update on the relationship of fish intake with prostate, breast, and colorectal cancers. Crit. Rev. Food Sci. Nutr. 2011, 51, 855-871. [CrossRef] [PubMed]

20. Shannon, J.; O’Malley, J.; Mori, M.; Garzotto, M.; Palma, A.J.; King, I.B. Erythrocyte fatty acids and prostate cancer risk: A comparison of methods. Prostaglandins Leukot. Essent. Fat. Acids 2010, 83, 161-169. [CrossRef] [PubMed]

21. Park, S.Y.; Wilkens, L.R.; Henning, S.M.; Le Marchand, L.; Gao, K.; Goodman, M.T.; Murphy, S.P.; Henderson, B.E.; Kolonel, L.N. Circulating fatty acids and prostate cancer risk in a nested case-control study: The Multiethnic Cohort. Cancer Causes Control 2009, 20, 211-223. [CrossRef] [PubMed]

22. Dahm, C.C.; Gorst-Rasmussen, A.; Crowe, F.L.; Roswall, N.; Tjønneland, A.; Drogan, D.; Boeing, H.; Teucher, B.; Kaaks, R.; Adarakis, G.; et al. Fatty acid patterns and risk of prostate cancer in a case-control study nested within the European Prospective Investigation into Cancer and Nutrition. Am. J. Clin. Nutr. 2012, 96, 1354-1361. [CrossRef] [PubMed]

23. Chua, M.E.; Sio, M.C.; Sorongon, M.C.; Dy, J.S. Relationship of dietary intake of omega-3 and omega-6 Fatty acids with risk of prostate cancer development: A meta-analysis of prospective studies and review of literature. Prostate Cancer 2012, 2012, 826254. [CrossRef] [PubMed]

24. Brasky, T.M.; Darke, A.K.; Song, X.; Tangen, C.M.; Goodman, P.J.; Thompson, I.M.; Meyskens, F.L., Jr.; Goodman, G.E.; Minasian, L.M.; Parnes, H.L.; et al. Plasma phospholipid fatty acids and prostate cancer risk in the SELECT trial. J. Natl. Cancer Inst. 2013, 105, 1132-1141. [CrossRef] [PubMed]

25. Torfadottir, J.E.; Valdimarsdottir, U.A.; Mucci, L.A.; Kasperzyk, J.L.; Fall, K.; Tryggvadottir, L.; Aspelund, T.; Olafsson, O.; Harris, T.B.; Jonsson, E.; et al. Consumption of fish products across the lifespan and prostate cancer risk. PLoS ONE 2013, 8, e59799. [CrossRef] [PubMed]

26. Pelser, C.; Mondul, A.M.; Hollenbeck, A.R.; Park, Y. Dietary fat, fatty acids, and risk of prostate cancer in the NIH-AARP diet and health study. Cancer Epidemiol. Biomarkers Prev. 2013, 22, 697-707. [CrossRef] [PubMed]

27. Dougherty, R.M.; Galli, C.; Ferro-Luzzi, A.; Iacono, J.M. Lipid and phospholipid fatty acid composition of plasma, red blood cells, and platelets and how they are affected by dietary lipids: A study of normal subjects from Italy, Finland, and the USA. Am. J. Clin. Nutr. 1987, 45, 443-455. [CrossRef] [PubMed]

28. Moreel, X.; Allaire, J.; Leger, C.; Caron, A.; Labonté, M.È.; Lamarche, B.; Julien, P.; Desmeules, P.; Têtu, B.; Fradet, V. Prostatic and dietary omega-3 fatty acids and prostate cancer progression during active surveillance. Cancer Prev. Res. 2014, 7, 766-776. [CrossRef] [PubMed]

29. Epstein, J.I.; Zelefsky, M.J.; Sjoberg, D.D.; Nelson, J.B.; Egevad, L.; Magi-Galluzzi, C.; Vickers, A.J.; Parwani, A.V.; Reuter, V.E.; Fine, S.W.; et al. A Contemporary Prostate Cancer Grading System: A Validated Alternative to the Gleason Score. Eur. Urol. 2016, 69, 428-435. [CrossRef] [PubMed]

30. Epstein, J.I.; Egevad, L.; Amin, M.B.; Delahunt, B.; Srigley, J.R.; Humphrey, P.A. The 2014 International Society of Urological Pathology (ISUP) Consensus Conference on Gleason Grading of Prostatic Carcinoma: Definition of Grading Patterns and Proposal for a New Grading System. Am. J. Surg. Pathol. 2016, 40, 244-252. [CrossRef] [PubMed]

31. Shaikh, N.A.; Downar, E. Time course of changes in porcine myocardial phospholipid levels during ischemia. A reassessment of the lysolipid hypothesis. Circ. Res. 1981, 49, 316-325. [CrossRef] [PubMed]

32. Da Silva, M.S.; Julien, P.; Bilodeau, J.F.; Barbier, O.; Rudkowska, I. Trans Fatty Acids Suppress TNF-alpha-Induced Inflammatory Gene Expression in Endothelial (HUVEC) and Hepatocellular Carcinoma (HepG2) Cells. Lipids 2017, 52, 315-325. [CrossRef] 
33. Labonte, M.E.; Cyr, A.; Baril-Gravel, L.; Royer, M.M.; Lamarche, B. Validity and reproducibility of a web-based, self-administered food frequency questionnaire. Eur. J. Clin. Nutr. 2012, 66, 166-173. [CrossRef] [PubMed]

34. Allaire, J.; Moreel, X.; Labonte, M.E.; Leger, C.; Caron, A.; Julien, P.; Lamarche, B.; Fradet, V. Validation of the omega-3 fatty acid intake measured by a web-based food frequency questionnaire against omega-3 fatty acids in red blood cells in men with prostate cancer. Eur. J. Clin. Nutr. 2015, 69, 1004-1008. [CrossRef] [PubMed]

35. Godin, G. The Godin-Shephard leisure-time physical activity questionnaire. Health Fit. J. Can. 2011, 4, 18-22.

36. Amireault, S.; Godin, G. The Godin-Shephard leisure-time physical activity questionnaire: Validity evidence supporting its use for classifying healthy adults into active and insufficiently active categories. Percept. Mot. Skills 2015, 120, 604-622. [CrossRef] [PubMed]

37. Koelwyn, G.J.; Wennerberg, E.; Demaria, S.; Jones, L.W. Exercise in Regulation of Inflammation-Immune Axis Function in Cancer Initiation and Progression. Oncology (Williston Park) 2015, 29, 908-920, 922. [PubMed]

38. Ouellet, V.; Weisnagel, S.J.; Marois, J.; Bergeron, J.; Julien, P.; Gougeon, R.; Tchernof, A.; Holub, B.J.; Jacques, H. Dietary cod protein reduces plasma C-reactive protein in insulin-resistant men and women. J. Nutr. 2008, 138, 2386-2391. [CrossRef] [PubMed]

39. Ouellet, V.; Marois, J.; Weisnagel, S.J.; Jacques, H. Dietary cod protein improves insulin sensitivity in insulin-resistant men and women: A randomized controlled trial. Diabetes Care 2007, 30, 2816-2821. [CrossRef] [PubMed]

40. Kelavkar, U.P.; Hutzley, J.; Dhir, R.; Kim, P.; Allen, K.G.; McHugh, K. Prostate tumor growth and recurrence can be modulated by the omega-6:omega-3 ratio in diet: Athymic mouse xenograft model simulating radical prostatectomy. Neoplasia 2006, 8, 112-124. [CrossRef]

41. Akinsete, J.A.; Ion, G.; Witte, T.R.; Hardman, W.E. Consumption of high omega-3 fatty acid diet suppressed prostate tumorigenesis in C3(1) Tag mice. Carcinogenesis 2012, 33, 140-148. [CrossRef]

42. Lloyd, J.C.; Masko, E.M.; Wu, C.; Keenan, M.M.; Pilla, D.M.; Aronson, W.J.; Chi, J.T.; Freedland, S.J. Fish oil slows prostate cancer xenograft growth relative to other dietary fats and is associated with decreased mitochondrial and insulin pathway gene expression. Prostate Cancer Prostatic Dis. 2013, 16, $285-291$. [CrossRef] [PubMed]

43. Gevariya, N.; Besancon, M.; Robitaille, K.; Picard, V.; Diabaté, L.; Alesawi, A.; Julien, P.; Fradet, Y.; Bergeron, A.; Fradet, V. Omega-3 fatty acids decrease prostate cancer progression associated with an anti-tumor immune response in eugonadal and castrated mice. Prostate 2019, 79, 9-20. [CrossRef] [PubMed]

44. Liang, P.; Henning, S.M.; Schokrpur, S.; Wu, L.; Doan, N.; Said, J.; Grogan, T.; Elashoff, D.; Cohen, P.; Aronson, W.J. Effect of Dietary Omega-3 Fatty Acids on Tumor-Associated Macrophages and Prostate Cancer Progression. Prostate 2016, 76, 1293-1302. [CrossRef] [PubMed]

45. Demark-Wahnefried, W.; Polascik, T.J.; George, S.L.; Switzer, B.R.; Madden, J.F.; Ruffin, M.T.; Snyder, D.C.; Owzar, K.; Hars, V.; Albala, D.M.; et al. Flaxseed supplementation (not dietary fat restriction) reduces prostate cancer proliferation rates in men presurgery. Cancer Epidemiol. Biomarkers Prev. 2008, 17, 3577-3587. [CrossRef] [PubMed]

46. Freeman, V.L.; Meydani, M.; Hur, K.; Flanigan, R.C. Inverse association between prostatic polyunsaturated fatty acid and risk of locally advanced prostate carcinoma. Cancer 2004, 101, 2744-2754. [CrossRef] [PubMed]

47. Tayari, N.; Heerschap, A.; Scheenen, T.W.J.; Kobus, T. In vivo MR spectroscopic imaging of the prostate, from application to interpretation. Anal. Biochem. 2017, 529, 158-170. [CrossRef] [PubMed]

48. Kobus, T.; Vos, P.C.; Hambrock, T.; De Rooij, M.; Hulsbergen-Van de Kaa, C.A.; Barentsz, J.O.; Heerschap, A.; Scheenen, T.W. Prostate cancer aggressiveness: In vivo assessment of MR spectroscopy and diffusion-weighted imaging at 3 T. Radiology 2012, 265, 457-467. [CrossRef] [PubMed]

49. Bellomo, G.; Marcocci, F.; Bianchini, D.; Mezzenga, E.; D’Errico, V.; Menghi, E.; Zannoli, R.; Sarnelli, A. MR Spectroscopy in Prostate Cancer: New Algorithms to Optimize Metabolite Quantification. PLoS ONE 2016, 11, e0165730. [CrossRef] [PubMed]

50. Sciarra, A.; Panebianco, V.; Ciccariello, M.; Alfarone, A.; Gentilucci, A.; Parente, U.; Passariello, R.; Gentile, V. Magnetic resonance spectroscopic imaging (1H-MRSI) and dynamic contrast-enhanced magnetic resonance (DCE-MRI): Pattern changes from inflammation to prostate cancer. Cancer Investig. 2010, 28, 424-432. [CrossRef]

51. Kurhanewicz, J.; Vigneron, D.B. Advances in MR spectroscopy of the prostate. Magn. Reson. Imaging Clin. N. Am. 2008, 16, 697-710. [CrossRef] 
52. De Marzo, A.M.; Platz, E.A.; Sutcliffe, S.; Xu, J.; Grönberg, H.; Drake, C.G.; Nakai, Y.; Isaacs, W.B.; Nelson, W.G. Inflammation in prostate carcinogenesis. Nat. Rev. Cancer 2007, 7, 256-269. [CrossRef] [PubMed]

53. Sfanos, K.S.; De Marzo, A.M. Prostate cancer and inflammation: The evidence. Histopathology 2012, 60, 199-215. [CrossRef] [PubMed]

54. Ratnayake, W.M.; Galli, C. Fat and fatty acid terminology, methods of analysis and fat digestion and metabolism: A background review paper. Ann. Nutr. Metab. 2009, 55, 8-43. [CrossRef] [PubMed]

55. Davidson, J.; Rotondo, D.; Rizzo, M.T.; Leaver, H.A. Therapeutic implications of disorders of cell death signalling: Membranes, micro-environment, and eicosanoid and docosanoid metabolism. Br. J. Pharmacol. 2012, 166, 1193-1210. [CrossRef]

56. Nash, S.H.; Schenk, J.M.; Kristal, A.R.; Goodman, P.J.; Lucia, M.S.; Parnes, H.L.; Thompson, I.M.; Lippman, S.M.; Song, X.; Gurel, B.; et al. Association between Serum Phospholipid Fatty Acids and Intraprostatic Inflammation in the Placebo Arm of the Prostate Cancer Prevention Trial. Cancer Prev. Res. 2015, 8, 590-596. [CrossRef]

57. Lindberg, J.; Klevebring, D.; Liu, W.; Neiman, M.; Xu, J.; Wiklund, P.; Wiklund, F.; Mills, I.G.; Egevad, L.; Grönberg, H. Exome sequencing of prostate cancer supports the hypothesis of independent tumour origins. Eur. Urol. 2013, 63, 347-353. [CrossRef]

58. VanderWeele, D.J.; Brown, C.D.; Taxy, J.B.; Gillard, M.; Hatcher, D.M.; Tom, W.R.; Stadler, W.M.; White, K.P. Low-grade prostate cancer diverges early from high grade and metastatic disease. Cancer Sci. 2014, 105, 1079-1085. [CrossRef]

(C) 2019 by the authors. Licensee MDPI, Basel, Switzerland. This article is an open access article distributed under the terms and conditions of the Creative Commons Attribution (CC BY) license (http://creativecommons.org/licenses/by/4.0/). 